\title{
Altitude, négritude, bravitude ou la résurgence d'une suffixation
}

\author{
Koehl, Aurore \\ Université de Lorraine, ATILF, UMR 7118, Nancy, F-54000, France \\ Aurore.Koehl@atilf.fr
}

\section{Introduction ${ }^{1}$}

Le néologisme bravitude, cité dans diverses études, est à l'origine de la création de très nombreux noms en -itude. La renommée mondiale de ce néologisme n'a pourtant pas donné lieu à une étude morphologique approfondie sur la suffixation en -itude. Les études antérieures dédiées à cette suffixation sont basées sur les noms en -itude enregistrés dans les dictionnaires. Ces noms ne rendent pas compte du visage actuel du patron morphologique qui construit les noms désadjectivaux en -itude, bouleversé depuis la création de bravitude. Afin de témoigner des changements intervenus dans la construction des noms en -itude, nous proposons une étude morphologique et historique de cette suffixation. Notre étude est basée sur des données extensives, issues du journal Le Monde et de la Toile, qui témoignent des variations récentes de la suffixation en -itude. En mettant en lumière trois phases distinctes dans son développement, nous montrerons combien cette suffixation a subi une évolution particulière.

De prime abord, les noms en -itude ne se distinguent pas des autres noms désadjectivaux (e.g. banalité, grandeur, petitesse, franchise, niaiserie, discrétion, parallélisme) par un fonctionnement sémantique particulier. A l'instar des autres noms désadjectivaux, les noms en -itude peuvent renvoyer à une propriété physique (1) ou non physique (2).

(1) vastitude, grandeur, petitesse, parallélisme

[+ Physique $]$

(2) inquiétude, discrétion, niaiserie, franchise

[- Physique]

Toutefois, nous verrons que cet apriori est incorrect. L'hypothèse que nous défendons est que les noms en -itude se situent en périphérie du système de construction morphologique des noms désadjectivaux du français. Notre objectif est donc de mettre en évidence les éléments qui font la particularité de la suffixation en -itude.

Après avoir présenté les données sur lesquelles se base cette étude (§2), nous étudierons successivement les noms issus du TLFi (§3), du journal électronique Le Monde (\$4) et de la Toile (\$5). La dernière section présente une synthèse globale de l'évolution de la suffixation en -itude du latin au français contemporain, (§6). Cette analyse est réalisée selon le cadre théorique, descriptif et prescriptif de la morphologie lexématique tel qu'il a été exposé par Matthews (1974), Anderson (1992) et Fradin (2003), entre autres. Dans la suite de cet article, nous noterons les noms désadjectivaux suffixés sur le modèle Asuf $f_{\mathrm{N}}$ où $\left({ }_{\mathrm{N}}\right)$ signale la catégorie du lexème construit, (A) la base adjectivale et (suf) l'exposant formel de la règle. Par exemple, les noms désadjectivaux en -itude seront notés Aitude $_{\mathrm{N}}$.

\section{Données}

Les noms étudiés sont issus de trois types de sources : (i) un dictionnaire informatisé, i.e. le Trésor de la langue française informatisé (noté TLFi), (ii) un journal sur CDRom, i.e. Le Monde pour les années 1987, 1991, 1995, 1999 et (iii) La Toile, cf. Tableau 1. Les données provenant de la Toile ont été relevées entre 2007 et 2011. 


\begin{tabular}{ll}
\hline Source & Nombre de noms étudiés \\
\hline TLFi & $47(\mathrm{cf}$. Annexe 7.1) \\
Le Monde $(1987,1991,1995,1999)$ & 21 (cf. Annexe 7.2) \\
La Toile ${ }^{2}$ & $372(\mathrm{cf}$. Annexe 7.3) \\
\hline Total & 440 \\
\hline
\end{tabular}

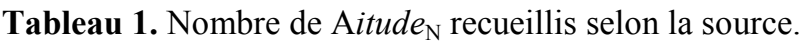

Seuls quarante-sept noms désadjectivaux en -itude sont issus du TLFi, ce qui est peu au regard des mille cent cinquante-deux noms désadjectivaux en -ité ou cent quatre-vingt-dix-huit noms désadjectivaux en -erie enregistrés par le même dictionnaire. Le corpus des noms étudiés est complété par vingt et une nouvelles formes recueillies dans le journal Le Monde et trois cent soixante-douze formes provenant de la

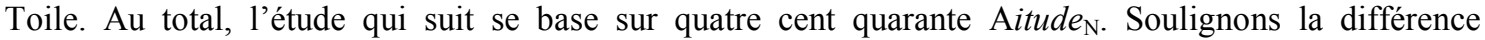
quantitative entre les données du TLFi et celles de la Toile, qui remet en cause le statut d'indisponibilité de la suffixation en -itude. En outre, il faut préciser que les données provenant de la Toile sont contextualisées, ce qui permet l'interprétation des nouvelles formes.

\section{Noms en -itude issus du TLFi}

\subsection{Origine latine et histoire de cette suffixation}

Parmi les noms construits relevés dans le lexique enregistré, il faut distinguer plusieurs cas. Les constructions françaises s'opposent aux emprunts au latin. Parmi les emprunts au latin, certains noms sont réanalysables en français (e.g. altitude, amplitude, etc.), d'autres pas (e.g. habitude, mansuétude). Nous avons conservé les noms construits en français et les noms empruntés au latin réanalysables en français (au moins par une partie des locuteurs). Quarante-sept noms désadjectivaux en -itude sont enregistrés dans le TLFi, dont vingt-trois sont issus du latin (3), par héritage naturel ou emprunt ultérieur (vocabulaire scientifique par exemple) (4)-(5), vingt-trois ne sont analysables que comme le résultat de constructions en français (6) cf. Darmesteter (1894), Nyrop (1908), Meyer-Lübke (1921) et Lüdtke (1978) et le dernier, FINITUDE, serait emprunté à l'anglais. Les informations étymologiques reportées ici, telles que la datation de la première attestation d'un lexème ou son origine, sont tirées des notices étymologiques du TLFi. La particularité de la recherche étymologique est qu'elle est conditionnée par la découverte des documents, ces informations sont donc à utiliser prudemment, comme des indications et non comme des vérités.

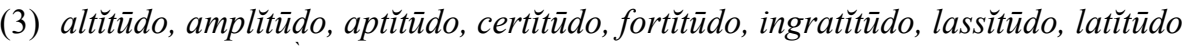

(4) $\operatorname{VASTITUDE}_{\mathrm{N}}\left(16^{\text {ème }}\right.$ siècle : emprunt au lat. vastǐtūdo «dévastation, ravage »; « proportions énormes », dér. de vastus)

(5) $\operatorname{PLANITUDE}_{\mathrm{N}}\left(18^{\mathrm{ème}}\right.$ siècle : emprunt au lat. tardif plānĭtūdo « surface plane »)

(6) $\operatorname{DECREPITUDE}_{\mathrm{N}}<\operatorname{DECREPIT}_{\mathrm{A}}\left(16^{\text {ème }}\right.$ siècle $) ; \operatorname{EXACTITUDE}_{\mathrm{N}}<\operatorname{EXACT}_{\mathrm{A}}\left(17^{\text {ème }}\right.$ siècle $)$

Parmi les créations françaises, neuf noms sont des corrélats négatifs des noms aptitude, certitude, correctitude, définitude, exactitude, finitude, quiétude et complétude :

(7) INAPTITUDE $<$ INAPTE $\left(14^{\text {ème }}\right.$ siècle $)$; INQUIETUDE $<$ INQUIET $\left(15^{\text {ème }}\right.$ siècle $)$

\subsubsection{Les noms de qualité latins en -tudo}

Meyer-Lübke (1921) note que l'héritage de la suffixation latine en -tudo est également rare dans la plupart des autres langues romanes. La perte de vitalité de cette suffixation daterait déjà de la période latine. En effet, Daude (2002), auteur d'une étude dédiée aux noms de qualité du latin, remarque que la vitalité de ce suffixe est surtout préclassique et décline vers la fin de la latinité. L'étude des noms de 
qualité latins de Daude comprend deux mille cent noms dont cent trente-sept noms en -tudo. Parmi ces noms en -tudo, nombreux sont ceux qui ont un concurrent satisfaisant davantage aux contraintes stylistiques. Voyons les quatre cas de concurrence relevés par Daude (2002 : 253) :

- Quatre-vingt-deux noms en -tudo ont un doublet en -tas. C'est la concurrence la plus fréquente parmi les noms de qualité latins, mais cette rivalité est plus apparente que réelle. Cette concurrence a en fait souvent lieu pour des raisons stylistiques telles que la rime.

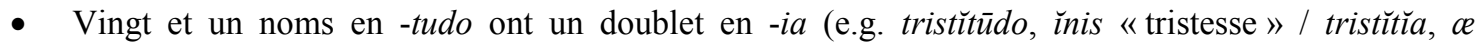
« tristesse »).

- Quinze noms en -tudo ont un doublet en -edo ou -ido de même sens (e.g. turpütūdo, ̌̆nis « laideur»/

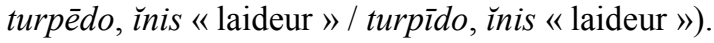

- Treize noms en -tudo ont subi une éclipse pendant la période classique car la langue classique semble leur préférer leurs concurrents en -tas, selon les observations de l'auteur.

Parallèlement à l'étude des concurrences formelles auxquelles sont soumis les noms de qualité latins, Daude examine les propriétés sémantiques de chaque suffixation impliquée dans la construction de ces noms. Il isole trois séries de noms en -tudo en fonction de leur sens. La première est celle des noms en -suētūdo, en lien avec la notion d'habitude. Nous n'avons relevé que quatre noms en -suētūdo dans le dictionnaire de Gaffiot (1934), reportés dans les exemples (8) à (11). Ces noms sont dérivés d'un adjectif, lui-même participe d'un verbe. Par exemple, le nom suètūdo aurait pour base l'adjectif suētus (a, um) « habitué, accoutumé ». Cet adjectif partage le radical supin du verbe intransitif suesco (suēvi, suētum, -ěre) « s'accoutumer, s'habituer ». La chaîne dérivationnelle est donnée pour chacun des quatre noms.

(8) SUETUDO, innis, f. « habitude, coutume » < SUETUS ( $a$, um) « habitué, accoutumé » < SUESCO « s'accoutumer, s'habituer»

(9) CONSUETUDO, ınis, f. «habitude, coutume, usage» < CONSUETUS (a, um) « habituel, accoutumé »< CONSUESCO « accoutumer »

(10) DESUETUDO, ı̌nis, f. « désaccoutumance, perte d'une habitude, désuétude » < DESUETUS ( $a$, um) « déshabitué » < dēsuesco « se déshabituer de, perdre l'habitude de »

(11) MANSUETUDO, ̌̌nis, f. « douceur, bonté, bienveillance » < MANSUETUS (a, um) « apprivoisé, doux »< MANSUESCO « apprivoiser »

Les deux premiers noms, SUETUDO et CONSUETUDO, sont synonymes, ce qui est surprenant, l'un étant la base morphologique de l'autre. Pourquoi créer une unité lexicale nouvelle dans ce cas ? La différence entre ces noms mérite d'être élucidée. Ernout \& Meillet (1959 : 663), dans leur dictionnaire étymologique de la langue latine, constatent la rareté de la série simple (SUESCO, SUETUS, SUETUDO). Le verbe SUESCO est surtout d'un usage poétique alors que ses dérivés sont usuels et classiques. Le nom SUETUDO serait apparu tardivement, reconstruit sur la forme CONSUETUDO. Le préfixe latin con-, utilisé dans la construction du verbe CONSUESCO, indique un procès arrivant à son terme (ibid. : 156). Ernout \& Meillet indiquent que " cette nuance de sens tend, du reste, à s'affaiblir et la forme à préverbe à se substituer à la forme simple, sans valeur spéciale », comme c'est le cas pour le couple CONSUESCO / SUESCO. La forme la plus courante, CONSUETUDO, a évolué pour donner en français le nom COUTUME. L'évolution phonologique 'naturelle' de la forme /tyd/ est la forme /tym/3 comme AMERTUME.

La deuxième série de noms en -tudo dégagée par Daude (2002) est constituée des noms dénotant une propriété physique observable / mesurable, cf. (12)-(13).

(12)ALBITUDO « blancheur »; VASTITUDO «proportions énormes »

(13)ALTITUDO « hauteur »; AMPLITUDO « ampleur »

La troisième série est formée des noms dénotant une qualité morale ou psychologique (14). Ces noms déterminent un comportement mesurable / perceptible.

(14)ÆGRITUDO « indisposition, chagrin »; ANXIETUDO « anxiété » 
En somme, les noms en -tudo du latin dénotent la propriété, physique ou non-physique et la mesure.

\subsubsection{Les noms en -itude en ancien français (avant 1330)}

Pour la période de l'ancien français, Tobler \& Lommatzsch enregistrent dans leur dictionnaire seulement dix-neuf noms en -(i)tude (que nous avons relevés grâce au Dictionnaire inverse de l'ancien français de Walker). Ces noms, retranscrits sous (15), dénotent une propriété.

(15)AMARITUDE 'amertume', BEATITUDE, CIRCUITUDE 'circonférence, contour', CONSUETUDE 'coutume', FORTITUDE, INGRATITUDE, LARGITUDE, LATITUDE, LONGITUDE, MANSUETUDE, MULTITUDE, PENITUDE, PLENITUDE, PRIMITUDE, RECTITUDE, SERVITUDE, SIMILITUDE, SOLICITUDE, SOLITUDE

Comment expliquer une telle perte de disponibilité ? Tout d'abord, il faut se rappeler qu'un très petit nombre de documents rédigés en ancien français est arrivé jusqu'à nous. Ensuite, il ne faut pas oublier que la majorité des textes qui nous sont parvenus présentent une forme versifiée et ne sont donc pas complètement représentatifs de la langue parlée à l'époque. Enfin, on observe le foisonnement des doublons à ce stade de la langue. Les alternances suffixales sont monnaie courante aux stades antérieurs de la langue française, pour satisfaire la rime ou le compte des pieds. Ces contraintes prosodiques rendent la forme -itude absente des textes versifiés, en raison de sa longueur et de la difficulté à trouver une rime en /tyd/. En effet, un nom en -itude ne peut guère rimer qu'avec le nom ETUDE ou un autre nom en -itude comme par exemple AMARITUDE et MULTITUDE en (16). Dans ces vers, AMARITUDE désigne « la qualité de ce qui est amer».

(16)a. Assez doulouse, assez lamente;

Mout se complaint, mout se demente,

Mout est en grant amaritude.

Ne vos sai pas la multitude

De sa tristrece racorder,

(Gautier de Coinci. Miracles de Notre-Dame, 1218, p. 54, II Mir.13, v. 297-301)

b. $\quad$ "Elle se plaint beaucoup, elle se lamente beaucoup; elle se plaint beaucoup, elle se désole beaucoup, elle est en très grand désespoir. Je ne sais pas vous rapporter l'étendue de sa tristesse »"

À l'encontre de ce qui a été observé pour les noms désadjectivaux en -eur et -esse (Koehl, 2010) pour la même période, peu de nouvelles formes en -itude ont été créées pour des raisons prosodiques. Au contraire, les contraintes prosodiques (rime, compte des pieds) propices au foisonnement de formes en -eur et -esse ont conduit, dans le même temps, à une faible représentation des formes en -itude dans les textes de cette époque.

La rareté des documents ayant traversé les siècles depuis le Moyen-âge, les contraintes formelles et la concurrence suffixale sont autant de difficultés semées sur le chemin de l'évolution de la suffixation en -itude.

\subsubsection{Les noms en -itude en moyen français (1330-1500)}

Pour la période du moyen français, le DMF (Dictionnaire du Moyen Français, version 2010) enregistre cinquante-six formes nominales en -(i)tude. En ancien et moyen français, comme en latin, les noms en -itude dénotent des propriétés. Ainsi, le nom ALTITUDE en moyen français pouvait renvoyer à la hauteur, à la manière de l'exemple (17). Il n'est plus vraiment possible pour le locuteur du français contemporain de parler de l'altitude d'un arbre. Le référentiel utilisé pour l'altitude n'est pas le même que celui utilisé pour la hauteur.

(17)Il n'a pas dix ans que au tres fructueux et opulent vignoble de Bourgonne flourissoit ung gros arbre de admirable altitude (MOLINET, Faictz Dictz D., 1467-1506, 232) 
Vingt et un noms en -itude relevés dans le DMF ne sont attestés ni en ancien français, ni en français contemporain. Ces formes sont des créations éphémères qui ont vu le jour dans le but de satisfaire aux contraintes prosodiques (cf. Merk, 1982), ou des termes scientifiques appartenant aux terminologies médicale (19) et religieuse (20) de l'époque. Le nom LARGITUDE « largesse » en (18) rime avec les noms PLENITUDE et FORTITUDE qui le suivent. Les formes LARGESSE, LARGETE, LARGEUR et LARGITION sont également enregistrées dans le DMF, avec le même sens, ce qui vient confirmer le choix de la forme en itude pour des raisons prosodiques. Ce choix, prohibé par la rédaction versifiée, ne pose aucun problème dans le cas d'une rédaction en prose.

(18) a. $\quad$ Bonté divine (...), Par largitude ottroie plenitude De fortitude a ta dolente ancelle Et prens pitié d'une povre pucelle. (MOLINET, Faictz Dictz D., 1467-1506, 80).

b. «Bonté divine (...), par largesse octroie une grande force à ta dolente servante et prends pitié d'une pauvre pucelle. »

(19)CONVALITUDE, EGRITUDE, EPAISSITUDE, LIMPITUDE, SALSITUDE, SPISSITUDE, VALITUDE

\section{(20)CELSITUDE PULCRITUDE, SAINTITUDE, SANCTITUDE}

On peut imaginer avoir relevé davantage de noms en -itude pour la période du moyen français par rapport à celle de l'ancien français en raison du plus grand nombre de textes nous étant parvenus pour le moyen français d'une part et par le changement de mode rédactionnel d'autre part.

Si les noms en -itude ont conservé une forme bien identifiable, héritée du latin, il s'agit de vérifier si les propriétés sémantiques des noms en -itude ont perduré du latin au français contemporain.

\subsection{Description sémantique des Aitude $_{\mathrm{N}}$ enregistrés dans le TLFi}

Dubois (1962), décrit les noms en -itude comme de simples noms de qualité, alors que deux groupes sémantiques semblent se dessiner dans le $T L F i$ autour de la date charnière de 1934 ; les noms scientifiques (\$3.2.1) et les noms d'appartenance à un groupe (humain) (\$3.2.2).

\subsubsection{Avant 1934 : des noms scientifiques (de mesure)}

Les noms en -itude enregistrés dans le TLFi et attestés avant 1934 présentent quelques particularités remarquables. Une partie de ces noms appartient au vocabulaire scientifique et dénote une mesure. Quelques exemples sont reportés dans le Tableau 2. Les noms de mesure en -itude, à l'instar de leurs bases adjectivales, renvoient à des propriétés physiques de dimension spatiale, et plus particulièrement à une échelle de mesure. Ces Aitude ${ }_{\mathrm{N}}$ sont les corrélats scientifiques de noms populaires en -eur (HAUTEUR, AMPLEUR, LONGUEUR, GRANDEUR, cf. Koehl 2010).

\begin{tabular}{|c|c|c|}
\hline Aitude $_{\mathrm{N}}$ & Définition donnée par le $T L F i$ & Origine donnée par le $T L F i$ \\
\hline ALTITUDE & $\begin{array}{l}\text { Hauteur d'un lieu ou d'un engin (avion, vaisseau } \\
\text { spatial...) mesurée par rapport au niveau de la mer }\end{array}$ & $\begin{array}{l}\text { Emprunt au lat. alť̈tūdo } \\
\text { « hauteur » }\end{array}$ \\
\hline AMPLITUDE & $\begin{array}{l}\text { Dans différents domaines scientifiques. Écart entre } \\
\text { deux points extrêmes d'un mouvement ou d'un } \\
\text { phénomène périodique }\end{array}$ & $\begin{array}{l}\text { Emprunt au lat. amplĭtūdo } \\
\text { «ampleur, largeur » }\end{array}$ \\
\hline LONGITUDE & $\begin{array}{l}\text { ASTRON. et GÉOGR. [Dans un système de } \\
\text { coordonnées] Angle de deux plans qui passent par } \\
\text { le pôle du système. }\end{array}$ & $\begin{array}{l}\text { Emprunt au lat. longĭtūdo } \\
\text { «longueur », dér. de longus } \\
\text { «long» }\end{array}$ \\
\hline MAGNITUDE & $\begin{array}{l}\text { ASTRON. Nombre indiquant l'éclat apparent d'un } \\
\text { astre et obtenu par divers procédés }\end{array}$ & $\begin{array}{l}\text { Emprunt au lat. magnĭtūdo } \\
\text { «grandeur, étendue » }\end{array}$ \\
\hline
\end{tabular}

Tableau 2. Un échantillon des noms de mesure scientifique en -itude, enregistrés dans le TLFi. 
La seconde partie des noms en -itude enregistrés dans le $T L F i$ et attestés avant 1934 renvoient à des propriétés physiques comme DECREPITUDE en (21), création savante utilisée en biologie par Ambroise Paré, ou non physiques (22) et à des états (23).
(21)DECREPITUDE
[Le compl. prép. désigne une pers., une partie du corps]
Dégradation physique accentuée produite par l'âge (1564)
(22)CERTITUDE
I. Vieilli, rare. [Correspond à certain ${ }^{2} \mathrm{~A}$ ] Assurance, garantie (> lat.)
II. Usuel. [Correspond à certain; les constr. ont pour base une phrase du type qqn a la certitude de... que... ou qqn est certain de..., que]
(23)SERVITUDE état de celui qui est serf ( $>$ lat.)

Le patron morphologique $\mathrm{A}>\mathrm{A}_{\text {itude }}$ utilisé en français jusqu'au dix-neuvième siècle est calqué sur le patron latin qui construisait des noms de propriété en -tudo. En latin, les noms en -tudo pouvaient recouvrir tous les sens de l'adjectif base, comme les noms en -itude aux stades d'ancien et de moyen français. Ce n'est plus le cas des noms en -itude enregistrés dans TLFi et attestés avant 1934. En effet, les noms en -itude que nous venons de passer en revue ne dénotent pas systématiquement une propriété. Par

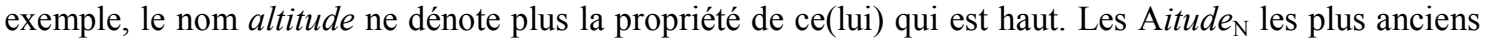
renvoient pour la plupart à des notions scientifiques, le plus souvent pour nommer une mesure, cf. Tableau 2. Les premiers indices du lien entre vocabulaire savant et suffixation en -itude émergent en moyen français, cf. les séries (19)-(20). Ces noms désadjectivaux savants se distinguent sémantiquement des noms les plus récents enregistrés dans le TLFi. La section suivante expose les évolutions sémantiques subies par cette suffixation après la date charnière de 1934.

\subsubsection{Après 1934 : des noms d'appartenance à un groupe (ethnique)}

En 1934, Aimé Césaire dans le journal l'Etudiant Noir crée la notion de NEGRITUDE, cf. (24). Ce terme devient le nom d'un mouvement littéraire, fondé par A. Césaire et L. Sédar Senghor.

(24)« La Négritude est la simple reconnaissance du fait d'être noir, et l'acceptation de ce fait, de notre destin de Noir, de notre histoire et de notre culture » (l'Etudiant Noir, 1934)

Cette notion est très largement médiatisée et le patron Aethnique $>_{\text {-itude }} \mathrm{N}$ est rapidement repris par d'autres mouvements de revendication d'une identité ethnique, ou pouvant être considérée comme telle. Ainsi, le $T L F i$ enregistre six autres noms d'appartenance à un groupe social opprimé. Les exemples (25) à (27) rapportent les définitions de ces sept noms.

(25) a. NEGRITUDE

b. Ensemble des valeurs propres aux cultures et civilisations des peuples de race noire; appartenance à cette race $[T L F i]$

(26) a. CORSITUDE, FRANCITUDE, FEMINITUDE, MAIGRITUDE, QUEBECITUDE

b. Des dér. en -itude sont créés à partir d'ethniques ou d'adj. exprimant l'appartenance à un groupe. [...] -itude tend à exprimer l'état d'oppression, d'aliénation. Le point de départ paraît être négritude (ca 1933). J. Bourquin relève corsitude, féminitude, maigritude, québécitude $[T L F i]$

(27) a. FEMELLITUDE

b. Néologisme, formé à partir de femelle, à l'image du terme négritude. Désigne, sous la plume de Xavière Gauthier, qui lui a donné naissance, l'ensemble des élans, des habitudes, des réactions affectives, des inhibitions et des interdits qui caractérisent « les femmes » prises en tant qu'ensemble social pensant et isolé du reste de la société (GIRAUD-PAMART Nouv. 1974) [TLFi]

Les définitions fournies par le TLFi s'appuient sur les travaux de Giraud et al. (1974), Ravier (1978) et Bourquin (1979). Outre l'appartenance à un groupe (ethnique), ces noms symbolisent l'oppression des membres de ce groupe. Dans les énoncés (28)-(29), nous avons souligné les éléments qui marquent la servitude. En (28) le syntagme au sang noir interdite renvoie à l'interdiction d'accès à certains milieux et 
lieux publics et l'image du cordon de police marque l'exclusion de la population noire. Dans l'extrait (29), l'oppression est explicitement évoquée, tout comme la perte de l'identité.

(28)ignorez les restaurants et les piscines, et la noblesse au sang noir interdite Et la Science et l'Humanité, dressant leurs cordons de police aux frontières de la négritude. (SENGHOR L. S., 1948, Hosties noires, 83-84)

(29)À partir de nos valeurs d'opprimées, faire jaillir nos valeurs d'avenir. La Féminitude peut-être. Comme pour la Négritude, renouer avec l'identité perdue. (HALIMI G., 1992, La Cause des femmes, 20-23)

L'observation des noms en -itude contenus dans le $T L F i$ met au jour que vingt-trois de ces noms sont des noms savants empruntés au latin. À partir du seizième siècle, de nouvelles unités, construites en français, rallient le stock latin. D'autres noms de propriété sans concurrent (LASSITUDE, PLATITUDE, PLANITUDE) se joignent au lexique français, ce qui indique la résistance de ce patron morphologique. Enfin, le vingtième siècle voit apparaître des noms renvoyant à «l'identité d'une ethnie péjorée », selon la description de Bourquin (1979: 57).

\section{Noms en -itude issus du journal Le Monde}

Vingt et une nouvelles formes, c'est-à-dire non enregistrées dans le TLFi, ont été relevées dans le journal Le Monde (pour les années 1987, 1991, 1995 et 1999). Ce nombre est non négligeable au regard des quarante-sept noms enregistrés dans le $T L F i$. Dans la continuité des noms exprimant l'identité d'une ethnie péjorée relevés dans le $T L F i$, la plupart des noms relevés dans le journal Le Monde traduisent une identité revendiquée, brandie comme un étendard, une fierté (\$4.1). Les autres noms, qui dénotent des attitudes, annoncent un nouveau type sémantique des Aitude $e_{\mathrm{N}}$ très répandu en français contemporain $(\S 4.2)$.

\subsection{L'identité revendiquée}

Sur le modèle de NEGRITUDE et dans la continuité de CORSITUDE, FRANCITUDE et QUEBECITUDE, treize des vingt et un nouveaux noms en -itude relevés dans Le Monde ont pour base un adjectif ethnique ${ }^{5}$. Dans leur contexte d'apparition abonde un vocabulaire qui établit le lien entre un groupe identitaire et l'oppression. En (30), les verbes parquer et contaminer marquent l'exclusion d'une minorité par la population.

(30)On nous a parqués dans des camps entourés de barbelés, dans des bâtisses en contreplaqué, loin des villes, sans doute afin que notre "arabitude" ne contamine pas la population, écrira Abdelkader Aïnine (Le Monde 1991)

Une fois le peuple sorti de l'oppression, se pose la question de la définition de son identité ethnique. Dans les extraits reproduits en (31)-(32), les noms BASQUITUDE et BELGITUDE renvoient à l'appartenance à un peuple, mais cette fois à un peuple qui n'est pas opprimé, contrairement à ARABITUDE en (30). Enfin, l'appartenance ethnique peut être revendiquée avec fierté. C'est le cas de l'emploi de CELTITUDE en (33). La revendication de l'identité celte est marquée dans cet exemple par le verbe PROCLAMER qui témoigne d'un sentiment de fierté.

(31)La démarche est aujourd'hui tout autre. Il ne s'agit plus de défendre une identité contestée, mais bien de réfléchir à l'usage qui sera fait de l'autonomie récemment recouvrée. Les organisateurs de ce deuxième congrès mondial veulent s'interroger sur ce qu'est aujourd'hui réellement la "basquitude" et sur les solutions à apporter à la profonde "crise de société" que connait aujourd'hui le Pays basque. (Le Monde 1987)

(32)Si le dernier livre de Pierre Mertens rencontre le succès que l'on connaît, c'est, outre le talent de son auteur, parce qu'il nous touche dans une de nos interrogations, qu'il nous incite à réfléchir à la belgitude et donc bien sûr à nos symboles. (Le Monde 1995) 
(33)Proclamant sa celtitude, Trystan Mordrel est le fils d'Olier Mordrel, fondateur en 1927 du Parti autonomiste breton, (Le Monde 1987)

De la NEGRITUDE à la CELTITUDE, il faut remarquer la perte du sens de servitude. Peut-être que la mode a été petit à petit moins à déplorer un état de servitude qu'à glorifier une situation (peuple combattant, aspiration à la liberté, revendication des droits, fierté des coutumes, etc.).

Comme le laissaient présager les Aitude $_{\mathrm{N}}$ du Monde, ceux recueillis en ligne ne sont plus seulement limités à l'oppression d'un peuple, mais étendus à l'expression de l'identité (culturelle) (34)-(35).

(34)a. $\quad$ ALSACIANITUDE $<$ ALSACIEN

b. L'homme le plus célèbre d'Alsace est aussi le plus incroyable des bosseurs. Voilà quarante ans et plus qu'il décline son alsacianitude dans tous les azimuts. ${ }^{6}$

(35)a. $\quad$ CAJUNITUDE < CAJUN

b. En Louisiane, la musique cajun n'a jamais été aussi populaire. Mais l'air ne fait pas la chanson, et la "cajunitude" peut être une cage

La construction de noms d'appartenance identitaire s'est également étendue à l'identité régionale, comme l'illustre par exemple le nom BRESSANITUDE, relevé sur la Toile cf. (36).

(36)Et elle revendique fort sa "bressanitude" qu'elle porte avec exemple et bonheur dans ses activités $^{8}$

\subsection{L'émergence des noms d'attitude}

Parallèlement à l'explosion des noms d'appartenance ethnique, six autres noms provenant du journal $L e$ Monde témoignent de la naissance de noms d'attitude en -itude (BRANCHITUDE, GARCITUDE, MALITUDE, PUNKITUDE, SINCERITUDE, VERTITUDE). Ces noms ont pour base un lexème qui renvoie à une qualité. Les noms GARCITUDE et VERTITUDE par exemple ne renvoient pas à une identité, encore moins à l'appartenance à un groupe assujetti, mais à un comportement (37)-(38)Erreur ! Source du renvoi introuvable., à une propriété sociale (revendiquée) (39)-(40).
a. $\quad$ trois femmes [...] trois adeptes de la garcitude
(Le Monde 1995)
b. = Trois garces, trois femmes qui se comportent comme des garces.
c. ? Trois femmes (très) garces, qui ont une attitude garce.

Ecologie qui m'a énervée... Peut-être aussi ne peut-on vivre éternellement loin de sa famille d'origine ? La question se poserait alors plutôt ainsi : "Pourquoi viens-tu si tard ?" Cette longue rupture se justifie par de multiples raisons. Quitte à jouer encore une fois les "vieilles taupes", il faut, pour l'expliquer, remonter aux aurores de la vertitude. (Le Monde 1991)

(39) a. la branchitude de Daft Punk

b. = Daft Punk est branché

(40) a. la mâlitude du commentateur

b. = Le commentateur mâle, qui a une attitude mâle.

c. $\quad=$ Le commentateur se comporte comme un mâle.

d. = Le commentateur revendique son appartenance au groupe mâle.

Ces $\mathrm{Aitude}_{\mathrm{N}}$ sont sémantiquement distincts des Aitude $\mathrm{N}_{\mathrm{N}}$ issus du TLFi. Ils font partie d'une longue série de noms d'attitude, ce que nous verrons dans la section 5.

Morphologiquement, on peut également supposer que ces noms résultent d'une autre règle qui contracte deux lexèmes bases pour former un mot-valise (cf. Grésillon, 1984 ; Fradin, 1997 ; Bat-El, 2006). Le deuxième lexème serait ATTITUDE. En (41), l'hypothèse d'une construction à partir d'ATTITUDE est possible.

$$
(41) \text { GARCE }_{\mathrm{A} / \mathrm{N}}, \operatorname{ATTITUDE}_{\mathrm{N}}>\text { GARCITUDE }_{\mathrm{N}}
$$$$
\text { « attitude (d'une) garce » }
$$ 
D'un côté, la coexistence de noms composés ou de ce qui s'y apparente (ZEN-ATTITUDE) et de noms en -itude (ZENITUDE) serait un argument en faveur de la formation d'un mot-valise. De l'autre, le parallèle entre ces noms (42) et des noms anciens comme INGRATITUDE (43), qui partagent le même sens construit, serait un argument en faveur de la suffixation en -itude. Bien que cette question soit intéressante, la nature morphologique de ces noms ne change rien à ce jeu de mots entre la forme du nom Xitude $\mathrm{N}_{\mathrm{N}}$ et le sens de ce nom « attitude $\mathrm{X} »$

(42)la garcitude de ces trois femmes

(43)l'ingratitude de ces trois femmes

Deux formes relevées dans Le Monde et absentes du TLFi restent à étudier : ESCLAVITUDE et MIXITUDE. La première provient d'une citation en langue ancienne, synonyme de SERVITUDE (44), que nous écartons de cette étude. La seconde est sémantiquement reliée aux noms d'appartenance ethnique, comme l'indiquent les sept adjectifs ethniques soulignés dans la citation (45).

(44) Voici la réponse du duc de Guise : « [...] il est aussy tost libre et hors de toute esclavitude [...]» (Le Monde, 1987)

(45)la table ronde autour de cette ville des confins a permis de mettre en évidence la richesse culturelle de toute une région exemplaire de mixitude, cette Bucovine d'abord moldave et ottomane, ensuite autrichienne et roumaine, enfin soviétique et ukrainienne mais toujours foyer de spiritualité juive. (Le Monde, 1999)

Les nouvelles formes issues du journal Le Monde dénotent soit l'appartenance à une identité revendiquée, soit l'attitude. Dix ans plus tard, quel reflet du patron morphologique $\mathrm{A}>\mathrm{Aitude} \mathrm{N}_{\mathrm{N}}$ renvoie la Toile?

\section{Noms en -itude issus de La Toile}

Une liste de candidats potentiels a été générée automatiquement à partir de la liste des adjectifs du TLFi. Les candidats ainsi générés ont ensuite été recherchés sur la Toile par le robot WaliM (Namer, 2003) via le moteur de recherche $\mathrm{Yahoo}^{\mathrm{TM}}$. Deux mille sept cent quarante candidats à la néologie apparaissent au moins une fois en ligne. Nous n'avons dépouillé que vingt pour cent des résultats non-nuls rapportés par le robot WaliM (soit cinq cent cinquante-neuf formes) sélectionnés au hasard. Ces résultats ont été triés pour éliminer les scories. Le tri consiste à éliminer les noms déverbaux, comme COUPITUDE sur COUPER en (46), ou dénominaux, comme CAROTTITUDE dérivé de CAROTTE en (47), les résultats pour lesquels le contexte est inexistant ou ne permet pas la compréhension de la forme en -itude, comme le nom CAMPITUDE en (48) pour lequel la base est ininterprétable, les noms apparaissant dans des travaux de linguistes tel que BOSNIANITUDE en (49), etc.

(46)j'aime beaucoup beaucoup même si de prime abord j'ai trouvé la coupitude de la tetê assez violente. ${ }^{9}$

(47)Certes, mais qui a déjà entendu, une carotte ou un poireau, fatigué et miné par un quotidien à faciès blafard, se plaindre de sa carottitude ou de sa poireausité ? ${ }^{10}$

(48)Les mannequins, au sommet de leurs campitude, se mêlèrent gaiement aux personnages du set. ${ }^{11}$

(49)bosnianitude ${ }^{12}$

Les exemples d'hésitations entre Aitude $\mathrm{N}_{\mathrm{N}}$ et composé en ATTITUDE sont fréquents sur la Toile, cf. (50).

(50)Et ca, c'est un probléme facile à résoudre. Suffit de voter des lois contre le racisme. Des lois sévéres. Genre "tirer à vue" au moindre signe de "nazitude" ou "nazi attitude"... ${ }^{13}$

Finalement, trois cent soixante-douze noms en -itude ont été conservés à partir de cet échantillon. Quantitativement et qualitativement, le décalage, lié à un événement de la vie politique française saute aux yeux (\$5.1). Les nouveaux noms en -itude sont souvent créés en écho à cet événement $(\$ 5.2)$ et dénotent toutes sortes de propriétés $(§ 5.3)$. 


\subsection{Le rôle de la médiatisation}

Le nombre de constructions de noms en -itude a explosé dans les productions des locuteurs ces cinq dernières années. Cet accroissement soudain de Aitude $_{\mathrm{N}}$ a été provoqué par la (sur)médiatisation d'une forme, BRAVITUDE, créée par une personnalité politique en période électorale. Le 6 janvier 2007, Ségolène Royal prononçait ces mots :

(51)« Comme le disent les Chinois, qui n'est pas venu sur la Grande muraille n'est pas un brave. Qui va sur la Grande Muraille conquiert la bravitude »

Cette forme néologique a engendré différents travaux, notamment sociolinguistiques. Rey (2008), par exemple, note le caractère pompeux de la formation, dans un ouvrage dédié au lexique politique de la campagne présidentielle de 2007 et à la récupération de ce lexique par les médias. Arrivé (2009) revient sur les aspects linguistiques, politiques et sociologiques de cette création. Pour lui, le nom BRAVOURE, synonyme de «courage, vaillance », est trop belliqueux et ne convenait pas pour exprimer l'idée de S. Royal. Il fallait un autre terme, correspondant au BRAVE «honnête, loyal ». En écho à la création de BRAVITUDE, Arrivé (2009) note trois types de réactions, politiquement orientées :

- L'acceptation du néologisme (E. Balladur, J. Lang);

- La condamnation de l'incompétence de S. Royal (Ac. M. Druon, A. Bentolila) ;

- La condamnation de l'attaque portée au français par S. Royal (L. Jospin).

Enfin, Arrivé (2009) cherche une cause sociologique à la violence de ces réactions. Il n'ose pas clairement imputer la virulence des reproches au fait que S. Royal soit une femme, mais souligne que dans le même temps, les formations du candidat N. Sarkozy (FATITUDE pour FATUITE ${ }^{14}$, HERITATION pour HERITAGE $^{15}$, PEREIN pour PERENNE, TRENTAGENAIRE pour TRENTENAIRE, CONQUERANCE pour CONQUETE) ou de L. Fabius ( « la courtesse des idées du Président ${ }^{16}$ ) ne suscitèrent pas le même émoi. Ainsi, Pierre Vandeginste, journaliste scientifique, à relevé 30 fois plus de bravitude que de fatitude sur la Toile pendant la période de la campagne présidentielle de 2007.

Quelle que soit la cause linguistique de ce néologisme, la citation a été relayée par la presse (journaux, radios) et par d'autres médias (e.g. blogs et forums politiques). P. Vandeginste explique que la création de bravitude, d'abord reprise exagérément par les médias, a reçu de nombreux échos de la part des utilisateurs de blogs et de forums (52).

(52)« la blogosphère, en l'occurrence, mime les distorsions de la réalité produites par la médiasphère $»^{17}$

Dans un article traitant de la relation entre langue et pensée, Feuillard (2009) aborde la néologie sous un angle sociolinguistique. Parmi les néologismes, l'auteur oppose les déviations aux innovations, par rapport à la norme institutionnelle. À propos des nouvelles unités comme bravitude, Feuillard (2009 : 158-159) parle de mise à l'index par la société «pour des raisons [qui ne sont pas] strictement linguistiques, notamment lorsqu'elles coexistent avec un terme déjà présent dans la langue ». Ce néologisme 'déviant', création lexicale sévèrement tancée par la classe politique qui fustige l'incompétence linguistique de la candidate et par conséquent son déficit de présidentialité, ce néologisme mis à l'index par la société et copieusement relayé par les médias a déclenché la création de très nombreux nouveaux noms en -itude étudiés dans la section suivante.

\subsection{Construire un Aitude $\mathrm{N}_{\mathrm{N}}$ pour répondre à un besoin de jeu}

De manière générale, la construction d'un Asuf $f_{\mathrm{N}}$ résulte du besoin d'un ancrage syntaxique nominal, c'est-à-dire que le sens de l'adjectif base est conservé mais que le locuteur/scripteur a syntaxiquement besoin d'une forme nominale. Dans le cas présent, l'objectif morphologique est uniquement de créer une forme en -itude et non plus de construire la forme nominale d'un adjectif, ni de construire un sens nouveau, cf. les travaux de Roché (à par.) et Sablayrolles (2000). En créant un nom en -itude, les utilisateurs de la Toile veulent faire référence à (51) ou simplement rendre leur énoncé amusant. Sur les 
trois cent soixante-douze résultats de la Toile, quarante et un Aitude ${ }_{\mathrm{N}}$ comprennent une référence explicite à la citation de Ségolène Royal.

\section{(53)Ségolène Royal, la connitude est de retour. ${ }^{18}$}

Cela place la règle de construction des noms en -itude dans un contexte sociolinguistique unique relativement aux autres constructions $\mathrm{A} s u f_{\mathrm{N}}$. De nouvelles formes sont construites par substitution de itude à une autre forme suffixale, ce qui produit un effet comique. En (54) par exemple, la forme COMPTABILITUDE est formée sur le nom COMPTABILITE par substitution de la forme -itude au suffixe -ité plutôt que construite sur l'adjectif COMPTABLE.

(54)[...] afin d'éviter que Ségo ne se mette en tête l'idée suicidaire de tenir seule sa comptabilitude.

La morphopragmatique, telle que définie par Dressler \& Merlini Barbaresi $(1987,1994,2001)$ et décrite par Merlini Barbaresi (2006) est

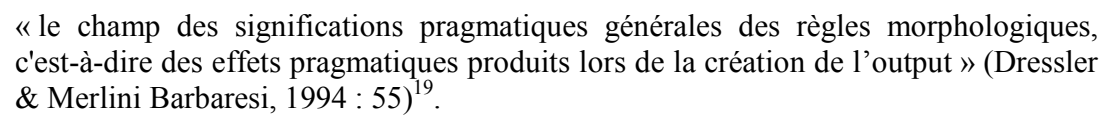

La règle de dérivation des noms en -itude relève de la morphopragmatique dans la mesure où elle contient une variable pragmatique qui ne peut être supprimée de la description du sens des nouveaux noms de propriété en -itude. Les formes relevées proviennent de blogs ou de forums. Dans une interaction entre un utilisateur de la Toile et son lecteur, un des obstacles à la communication est la distance qui sépare les interactants, ce qui les empêche d'entretenir une bonne relation interactionnelle. Les Aitude ${ }_{\mathrm{N}}$ relevés sont le produit d'utilisateurs de la Toile qui ont pour objectif de se rapprocher du lecteur, d'inclure le lecteur dans leur sphère et réciproquement de se faire admettre dans la sphère intime du lecteur afin de réduire la distance qui les sépare.

Bien des noms relevés sont des constructions éphémères, sémantiquement très éloignées des Aitude ${ }_{\mathrm{N}} \mathrm{du}$ $T L F i$. La citation en (51) fut si bien relayée par la machine médiatique que les locuteurs du français ont pu insuffler à ce patron morphologique un effet pragmatique comique.

\subsection{Les nouveaux Aitude ${ }_{\mathrm{N}}$ sont-ils des noms de propriété ?}

À l'instar des noms de qualité latins en -tudo décrits par Daude (2002) (cf. §3.1), les nouveaux noms en -itude relevés en ligne ont pour bases des adjectifs dénotant des propriétés physiques, telles que la dimension (55), la couleur (56) et des propriétés non-physiques parmi lesquelles se distinguent propriétés psychologiques (57) et comportementales (58).

(55) Et pour que vous preniez un peu mieux la mesure de la granditude du campus, je vous ai mis une photo ${ }^{20}$

(56) La jaunitude des feux de jours devient lassante ${ }^{21}$

(57) Que faire face à la tristitude du son, des images, des écrans ${ }^{22}$

(58) Le Royaume sombre définitivement dans la branquignolitude la plus totale. ${ }^{23}$

L'analyse des formations des scripteurs de la Toile nous permet d'affirmer que la formation de Aitude $_{\mathrm{N}}$ tient à la volonté d'utiliser -itude plutôt qu'à celle de nominaliser une propriété (adjectivale). De fait, aucune contrainte d'aucune sorte ne pèse sur la catégorie, le type morphologique de la base ou le sens. Enfin, l'utilisation du thème du féminin se généralise alors que les noms les plus anciens étaient construits sur le thème latin (cf. Plénat, 2008). 


\section{Synthèse globale}

\subsection{Une évolution originale grâce à la médiatisation de deux néologismes}

Ce panorama des constructions en -itude du français fait apparaître trois périodes dans l'histoire de la suffixation en -itude, cf. Figure 1. Cette suffixation a longtemps été représentée dans le lexique français par des noms savants issus du latin. Quelques constructions françaises ont vu le jour, mais ces nouveaux noms sont soit des noms savants, soit des noms construits sur des adjectifs préfixés par in- dont le corrélat positif A est nominalisé par la RCL-itude (e.g. INAPTITUDE). Les nouvelles formes ayant vu le jour avant le vingtième siècle sont en quantité négligeable. Les historiens de la langue et les morphologues considéraient cette suffixation indisponible, à juste titre, de la fin de la latinité jusqu'à il y a encore cinquante ans. C'était sans compter sur la médiatisation de deux nouvelles formes suffixées par -itude, la NEGRITUDE et la BRAVITUDE. Le premier de ces termes, très bien accueilli, a donné lieu à une multitude de noms d'appartenance ethnique. Le second, très critiqué, a ouvert la voie à la création de nombreux noms désadjectivaux intégrant un effet morphopragmatique comique. La contrainte sémantique limite le nombre de nouveaux noms ethniques car le nombre d'adjectifs ethniques est lui-même limité. L'absence de contrainte a l'effet inverse pour les noms en -itude répondant à un besoin de jeu. Le nombre de nouvelles formes en -itude est dans ce cas illimité.

\begin{tabular}{|c|c|c|c|}
\hline Nscientifique & Nethnique & Nattitude & Npropriété \\
\hline & 1934 & & 2007 \\
\hline
\end{tabular}

Figure 1. Évolution de la suffixation de -itude en français.

Parallèlement, les noms relevés dans le journal Le Monde laissent entrevoir un nouveau modèle de construction de noms d'attitude, cf. (37)-(43). Ces trois vagues successives posent la question du nombre de suffixations en -itude du français.

\subsection{Combien de suffixes -itude en français contemporain ?}

La coexistence de ces diverses constructions sème le trouble quant à la définition de la (ou des) suffixation(s) en -itude en français contemporain. Doit-on distinguer une, deux ou trois suffixations ? La suffixation en -itude a subi des modifications dans les trois niveaux de description lexématique qui sont les dimensions formelle, syntaxique et sémantique. Au niveau phonologique, on remarque que le thème adjectival sélectionné n'est plus le radical latin mais le radical qui sert à former le féminin. Au niveau syntaxique, la suffixation en -itude s'appliquait initialement à des adjectifs, puis la sélection des bases a été étendue aux noms (toponymes) pour la création de noms d'appartenance ethnique. Depuis 2007, on rencontre des noms en -itude construits sur des adjectifs, des noms, des verbes et des noms propres. Au niveau sémantique, le patron $\mathrm{A}>\mathrm{A}_{\mathrm{itude}} \mathrm{N}$ 'applique à des adjectifs pour créer des noms scientifiques (observation/mesure). La seconde vague de suffixation en -itude, initiée par Aimé Césaire, sélectionne des adjectifs ethniques (ARABE) ou des toponymes puis des adjectifs dénotant l'appartenance à un groupe social ou régional. Parallèlement, le patron $\mathrm{A}>\mathrm{A}_{\text {itude }}$ permet de créer des noms d'attitude (ZENITUDE). La troisième vague de création impulsée par le néologisme de SR permet de construire un nom en -itude à partir d'adjectifs, de noms, de verbes, de noms propres, de syntagmes, etc. Les Aitude $\mathrm{N}_{\mathrm{N}}$ issus de la troisième vague dénotent la propriété, mais sont créés non pas pour des raisons sémantiques mais pour des raisons de jeu.

Les noms savants ne sont plus construits par le biais de la règle de construction de lexèmes en -itude. La forme suffixale -itude n'est plus associée au vocabulaire scientifique en raison de son potentiel humoristique. Les nouveaux noms de concept, de notion ou de mesure appartenant à la terminologie scientifique sont construits au moyen de la suffixation en -ité, comme les noms CIRCULARITE, POLARITE et 
PROPORTIONNALITE dans les exemples (59)-(61) (cf. Koehl, 2009). Parmi les nouvelles formes recueillies sur la Toile, seul le nom OBLONGITUDE désigne une mesure. Cette forme marginale est probablement issue d'une analogie avec LONGITUDE.

(59)Une circularité de 0,95

(60)La polarité du polymère

(61)La proportionnalité de la chaine tend vers 6

Les noms d'appartenance identitaire en -itude sont nombreux. La suffixation en -itude est en concurrence avec -ité et -erie, qui sont deux autres suffixes nominalisateurs d'adjectifs ethniques (62)-(65)

(62)On ne peut pas nier ce qu'on est pas, c'est comme si tu disais a un chinois qu'il nie sa nipponité24

(63)Au début, j'ai cru qu'il s'agissait d'une nouvelle nipponerie d'Okuba. ${ }^{25}$

(64) [...] mais plutôt pour analyser avec vous toute la nipponitude que comporte ce reproche [...] c'est un fondamental du comportement japonais ${ }^{26}$

(65)Enfin, ils se moquent aussi de Jin l'Américain en affirmant fièrement leur nipponitude totale à grand coup d'armures robotisées comme les japonais savent si bien le faire... ${ }^{27}$

Les exemples (62)-(65) montrent la distribution sémantique des trois suffixes. La règle de construction de lexèmes en -erie peut construire des noms d'événements et d'artefacts. La suffixation en -ité est neutre (Dal \& Namer 2008), NIPPONITE désigne simplement le fait d'être NIPPON, de nationalité japonaise. Une personne ayant du sang nippon dans les veines peut prétendre à la nipponité. Le nom NIPPONITUDE est différent, il fait référence à l'identité japonaise, à l'appartenance à cette culture. Il existe des noms en -ité comme IVOIRITE qui peuvent renvoyer à la revendication d'une identité, à un mouvement de libération. L'étude des noms de (la revendication de) l'appartenance à un groupe donné mérite être approfondie.

Les noms ethniques en -itude et les noms de propriété en -itude ne sont pas le résultat de l'application de RCL différentes. En effet, d'autres propriétés peuvent être revendiquées, tout ce qui est constitutif de l'identité de quelqu'un ou quelque chose.

(66)Les chauves sont partout, dans le sport, la politique, la culture... Ils assument parait-t-il désormais leur chauvitude la tête haute. ${ }^{28}$

(67) $\mathrm{Au}$ moyen-âge, le monde était peut-être barbare mais on avait du respect et de l'honneur. Aujourd'hui, notre "civilisitude", c'est finalement ce qui nous permet de bouffer devant les Africains qui crèvent de faim. ${ }^{29}$

En conclusion, l'analyse des noms en -itude montre que la formation des noms de propriété suit un cheminement qui passe outre la frontière des suffixes, des contraintes sur l'input et du sens construit. Nous avons montré le pouvoir de la médiatisation de deux créations dans le cadre politique. Cette suffixation, jugée indisponible il y a quelques années, a bénéficié d'un nouveau souffle grâce à une intense couverture médiatique. La forme -itude, facilement identifiable, n'est plus assimilée au vocabulaire savant mais à l'appartenance identitaire et à son effet comique.

\section{Références bibliographiques}

Anderson, S. R. (1992). A-Morphous Morphology. Cambridge: Cambridge University Press.

Arrivé, M. (2009). Un néologisme pris sur le vif : la bravitude. Neologica, 3.

Bat-El, O. (2006). Blends. In Encyclopedia of Language and Linguistics (2 ed.). Oxford: Elsevier.

Bourquin, J. (1979). Remarques sur la formation néologique récente de substantifs en -itude dérivés ou non. In Adola, Roseline et al. (eds) Néologie et lexicologie. Hommage à Louis Guilbert, Paris : Larousse, 47-66.

Collet, O. (2000). Glossaire et index critiques des æuvres d'attribution certaine de Gautier de Coincy. Genève : Droz.

Dal G. \& Namer F. (2010). French Property Nouns Toponyms or Ethnic Adjective: a case of base variation. In Wolfgang U. Dressler, Dieter Kastovsky, Hans Christian Luschützky and Rainer Franz (Eds.), Variation and 
Change in Morphology. Selected papers from the 13th International Morphology Meeting, Vienna February 2008, Amsterdam/Philadelphia: John Benjamins, 53-73.

Darmesteter, A. (1877). De la création actuelle des mots nouveaux et des lois qui la régissent. Paris : Vieweg.

Darmesteter, A. (1894). Traité de la formation des mots composés dans la langue française (2 ed.). Paris : Honoré Champion.

Daude, J. (2002). Les substantifs abstraits de qualité. In C. Kircher-Durand (Ed.), Grammaire fondamentale du latin (Vol. IX. La création lexicale : la formation des noms par dérivation suffixale, pp. 225-305). Leuven / Paris : Peeters.

[DMF] = Dictionnaire $d u$ Moyen Français, version 2010. ATILF - CNRS \& Nancy Université. http://www.atilf.fr/dmf

Dressler, W. U., \& Merlini Barbaresi, L. (1987). Elements of morphopragmatics. Duisburg: LAUD A194.

Dressler, W. U., \& Merlini Barbaresi, L. (1994). Morphopragmatics. Diminutives and Intensifiers in Italian, German and Other Languages. Berlin: Mouton de Gruyter.

Dressler, W. U., \& Merlini Barbaresi, L. (2001). Morphopragmatics of diminutives and augmentatives: on the priority of pragmatics over semantics. In I. Kenesei \& R. M. Harnish (Eds.), Perspectives on semantics, pragmatics, and discourse (pp. 43-58). Amsterdam: Benjamins.

Dubois, J. (1962). Étude sur la dérivation suffixale en français moderne et contemporain. Paris : Larousse.

Ernout, A. \& Meillet, A. (1959). Dictionnaire étymologique de la langue latine. Histoire des mots (4 ${ }^{\text {ème }}$ éd.). Paris : Klincksieck.

Feuillard, C. (2009). Langue et expérience : adéquation, variation, altération? La Linguistique, 45(2), 153-160.

Fradin, B. (1997). Les mots-valises : une forme productive d'existants impossibles? In Corbin D., B. Fradin, B. Habert, F. Kerleroux \& M. Plénat (eds) Mots possibles et mots existants, Vol. 1, Silexicales. Villeneuve d'Ascq : URA 382 - CNRS \& Université de Lille 3, 101-110.

Fradin, B. (2003). Nouvelles approches en morphologie. Paris : Presses Universitaires de France.

Gaffiot, F. (1934). Dictionnaire latin-français. Paris : Hachette.

Giraud, J., Pamart, P., \& Riverain, J. (1974). Les nouveaux mots "dans le vent". Paris : Larousse.

Grésillon, A. (1984). La règle et le monstre : le mot-valise. Tübingen : Max Niemeyer Verlag.

Koehl, A. (2009). Are French -ité suffixed nouns property nouns?, In Selected Proceedings of the 6th Décembrettes, ed. Fabio Montermini, Gilles Boyé, and Jesse Tseng, 95-110. Somerville, MA: Cascadilla Proceedings Project.

Koehl, A. (2010). Les noms de propriété adjectivale en -eur et -esse : un modèle évolutif original. In Franck Neveu, Valelia Muni Toke, Thomas Klingler, Jacques Durand, Lorenz Mondada et Sophie Prévost (Eds.), Congrès Mondial de Linguistique Française - CMLF'10, La Nouvelle-Orléans, 991-1007.

Lüdtke, J. (1978). Prädikative Nominalisierung mit Suffixen im französichen, katalanischen und spanischen. Tübingen: Niemeyer.

Matthews, P. H. (1974). Morphology, 2 edition, 1991. Cambridge: Cambridge University Press.

Merk, G. (1982). Les héritiers et les substituts du suffixe latin-tione en gallo-romania. Atelier de reproduction des thèses : Université de Lille 3.

Merlini Barbaresi, L. (2006). Morphopragmatics. In K. Brown (Ed.), Encyclopedia of Language and Linguistics (Second Edition ed., pp. 332-335). Oxford: Elsevier Ltd.

Meyer-Lübke, W. (1921). Historische Grammatik der französichen Sprache (2 ${ }^{\text {nd }}$ ed. Vol. 2: Wortbildungslehre). Heidelberg: Winter.

Namer, F. (2003). WaliM : valider les unités morphologiques par le Web. Silexicales, 3, 142-150.

Nyrop, K. (1908). Grammaire historique de la langue française (Vol. 3). Copenhaguen : Nordisk Forlag. 
Plénat, M. (2008). Le thème L de l'adjectif et du nom. In J. Durand, B. Habert \& B. Laks (Eds.), Actes du Congrès Mondial de Linguistique Française 2008 (pp. 1613-1626). Paris : Institut de Linguistique Française.

Ravier, X. (1978). Espace linguistique français, espace linguistique occitan. La Banque des mots, 15, 29-44.

Rey, A. (2008). Lexi-com'.De Bravitude à Bling-Bling (Vol. 1) : Fayard.

Roché, M. (à par). Propositions en morphologie lexicale. Carnets de grammaire.

Sablayrolles, J.F. (2000). La néologie en français contemporain. Examen du concept et analyse de productions néologiques récentes. Paris : Honoré Champion.

Tobler, A. \& Lommatzsch E. (1925). Altfranzözisches Wörterbuch. Berlin: Weidmann, puis Wiesbaden: Steiner.

$[T L F i]=$ Trésor de la Langue Française. (1971-1994). Paris : Gallimard.

Walker, D. C. (1982). Dictionnaire inverse de l'ancien français. Ottawa : Ed. de l'université d'Ottawa. http://people.ucalgary.ca/ dcwalker/Dictionary/dict.html\#database

\section{ANNEXES}

\subsection{Aitude $_{\mathrm{N}}$ issus du TLFi}

altitude, amplitude, aptitude, béatitude, certitude, correctitude, corsitude, décrépitude, exactitude, femellitude, féminitude, finitude, fortitude, francitude, inaptitude, incertitude, incorrectitude, indéfinitude, inexactitude, infinitude, ingratitude, lassitude, latitude, longitude, magnitude, maigritude, négritude, nullitude, planitude, platitude, plénitude, promptitude, québécitude, rectitude, servitude, similitude, solitude, sollicitude, vastitude, vilitude, complétude, désuétude, disquiétude, hébétude, incomplétude, inquiétude, quiétude.

\subsection{Aitude $_{\mathrm{N}}$ issus du Monde}

albanitude, anglitude, arabitude, basquitude, belgitude, branchitude, celtitude, esclavitude, garcitude, germanitude, lyonnitude, mixitude, mâlitude, norditude, polonitude, punkitude, serbitude, sincéritude, sinitude, slavitude, vertitude.

\subsection{Aitude $_{\mathrm{N}}$ issus de laToile}

abjectitude, aboutitude, abstrusitude, admirabilitude, adultitude, agrégitude, aigritude, aliénitude, alsacianitude, amicalitude, amorphitude, anglo-saxonitude, angoissitude, ashkénazitude, auburnitude, autrichianitude, autritude, avachitude, avaritude, barbaritude, bellitude, beuritude, bienheureusitude, blafarditude, blaireautitude, blanchitude, blêmitude, bohêmitude, bon enfantitude, bonnarditude, boréalitude, lèche-bottitude, boudinitude, bouffitude, bouillitude, bourguignonitude, bourrutitude, bourritude, boursitude, branquignolitude, bravitude, bravachitude, bressanitude, bretonnitude, bébêtitude, cafritude, cajunitude, capitalitude, carrossitude, cartonnitude, casanitude, censitude, chaleureusitude, chauvitude, cheapitude, chiantitude, chinoisitude, chipotitude, chiquitude, chnoquitude, choutitude, civilisitude, clairitude, classitude, clichitude, cocassitude, cochonnitude, collectivitude, comblitude, communautaritude, compactitude, compliquitude, comptabilitude, confinitude, conjointitude, connitude, contractitude, cordialitude, coréanitude, craspecitude, crispitude, croustillantitude, crutitude, crépitude, crétinitude, cultivitude, cynicitude, céléritude, cérébralitude, cévenolitude, dansabilitude, différentitude, dingotitude, diplômitude, disparatitude, diversitude, divinitude, divorcitude, drôlitude, duplicitude, débilitude, débraillitude, déchaînitude, déférentitude, déglinguitude, dégoûtitude, délinquantitude, démerditude, dépititude, désabusitude, désuetitude, dévastitude, enflitude, expansitude, exsanguitude, extra-terrestritude, ébaubitude, économitude, électoralitude, élégantitude, énormitude, étourditude, évolutivitude, fastochitude, fatiguitude, sans-filitude, fiscalitude, flagrantitude, flamanditude, flapitude, flavitude, flexibilitude, flétritude, foireusitude, folkitude, foncitude, forestitude, fouillitude, 
fourbitude, francophonitude, frappadinguitude, frilositude, frisitude, frisquitude, froiditude, froussarditude, félonitude, genevoisitude, gentillitude, glacitude, gnan-gnanitude, goulutitude, gourmanditude, granditude, gravitude, grinchositude, gringitude, grisâtritude, grognonitude, guillerettitude, géantitude, générositude, habilitude, harditude, honnêtitude, humblitude, hybriditude, hétérosexualitude, illogicitude, illustritude, imberbitude, imbitabilitude, immigritude, immobilitude, immédiatitude, impunitude, impérialitude, inactivitude, inadaptitude, incarnitude, incongruitude, inconsolabilitude, incorruptibilitude, increvabilitude, indigénitude, interloquitude, intégritude, irrésistibilitude, islamicitude, jalousitude, jaunassitude, jaunitude, jouassitude, jouissivitude, joyeusitude, kamikazitude, kitschitude, laiditude, lamentabilitude, larvitude, latinitude, lauréatitude, lentitude, lesbianitude, libritude, loufoquitude, lugubritude, lyonnaisitude, légalitude, magnanimitude, magyaritude, maigrichonitude, maladroititude, malinitude, manchotitude, marocanitude, marseillaisitude, massivitude, matinalitude, mauritude, mirotitude, mièvritude, mobilitude, modestitude, moellitude, moisitude, moititude, monotonitude, monstritude, monumentalitude, moralitude, morbiditude, morfalitude, mortitude, moutonnitude, mythomanitude, mécréantitude, métissitude, mûritude, nacritude, nanitude, nantitude, naufragitude, navritude, nazitude1, nazitude2, nervositude, nipponitude, nomaditude, nordaméricanitude, nulliparitude, néophytitude, oblongitude, ocritude, opposabilitude, orphelinitude, ouvertitude, paillettitude, palpitantitude, paranormalitude, parisianitude, parmitude, passivitude, peinarditude, penchitude, permissivitude, perpendicularitude, picarditude, placiditude, plasticitude, pleurnicharditude, plouquitude, pluralitude, plâtritude, poivritude, polaritude, politicarditude, polyglottitude, pompitude, postmodernitude, pourpritude, prestigiositude, progressivitude, propritude, protestantitude, protitude, provisoiritude, puritude, pénarditude, périodicitude, quelconquitude, rabatjoitude, racornitude, radicalitude, raffinitude, raisonnabilitude, rancitude, rarissimitude, rebellitude, redevabilitude, refoulitude, riquiquitude, risquitude, robustitude, rognitude, romanitude, romanesquitude, ronchonitude, ronditude, roublarditude, rougitude, rouillitude, régionalitude, sacritude, salinitude, saumâtritude, savantitude, savourositude, savoyarditude, scolaritude, scélérattitude, selectitude, senioritude, sensualitude, sexistitude, sicilianitude, sidéritude, sinistritude, slovénitude, socialistitude, sombritude, stabilitude, stupiditude, suffisantitude, surfaititude, syndicalitude, séductivitude, sérieusitude, sérénissimitude, sévéritude, tamoulitude, temporaritude, tenabilitude, terrestritude, terroristitude, timoritude, toquitude, totalitude, touffuitude, toulousainitude, toxicitude, tristitude, trépidantitude, undergrounditude, valaisanitude, validitude, vaudoisitude, velutitude, vespéralitude, victimitude, viditude, vieillitude, viergitude, vigilantitude, vikingitude, vivacitude, vénalitude, waterproofitude, xénophobitude, youpinitude, zenitude, zinzinitude.

\footnotetext{
${ }^{1}$ Je remercie Fiammetta Namer ainsi que les relecteurs anonymes qui ont contribué à l'amélioration de cet article.

${ }^{2}$ Relevés en 2010.

${ }^{3}$ Voir par exemple Bourquin (1979: 50).

${ }^{4}$ Pour la traduction du nom AMARITUDE en « affliction, désespoir », cf. Collet (2000:27).

5 Albanitude, ANGlitude, ARABitude, BASQuitude, BElgitude, Celtitude, Germanitude, lyonNitude, NORDITUDE, POLONITUDE, SERBITUDE, SINITUDE, SLAVITUDE

${ }^{6}$ http://www.alsace-visite-guidee.info/ami sif.htm

${ }^{7}$ http://www.lactualite.com/20070705 105210 2056\&PAGE=2

${ }^{8}$ http://www.jeanfrancoisdebat.fr/en toute intimite/

${ }^{9}$ http://forum.hardware.fr/hfr/Photonumerique/Photos/portraits-hors-studio-sujet $91380 . \mathrm{htm}$

${ }^{10}$ http://www.zipiz.com/kronik39.htm

11 http://www.prestigium.com/defiles/defile-john-galliano/femme-pret-a-porter-automne-hiver-2008-paris/johngalliano-fantastique,-a-un-detail-pres 15697.html

${ }^{12}$ http://w3.erss.univ-tlse2.fr/textes/pagespersos/plenat/Hathout\&alii07.pdf
} 
${ }^{13} \mathrm{http://www.yabiladi.com/forum/mein-kampf-protocole-sages-sion-2-3649553.html}$

${ }^{14}$ Le 18 avril 2007, France Inter, «Hélène Jouan, ne me prêtez pas une telle fatitude » cf. l'article de Libération : http://www.liberation.fr/politiques/010120041-apres-la-bravitude-de-royal-la-fatitude-de-sarkozy

${ }^{15}$ Le 15 janvier 2007, en visite dans une entreprise au Mont Saint-Michel

${ }^{16}$ Le 04 mai 2005, sur France Inter.

${ }^{17} \mathrm{http} / / /$ www.aietech.com/leblog/2007/5/4/hritation-trentagnaire-fatitude-conqurance.html

${ }^{18}$ http://www.el-dia.com/actualites-1139.php

${ }^{19}$ Morphopragmatics can be defined as the area of the general pragmatic meanings of morphological rules, that is, of the regular pragmatics effects produced when moving from the input to the output of a morphological rule » (Dressler \& Merlini Barbaresi, 1994, p. 55)

${ }^{20} \mathrm{http}: / / 184$-jours-a-berlin.blogspot.com/2009/03/i-want-to-break-free-but-free.html

${ }^{21} \mathrm{http} / / /$ www.grioo.com/info5895.html

${ }^{22}$ http://www.blogbang.com/posts/246005-la-france-en-a-marre-de-la-tristitude

${ }^{23}$ http://www.etiennefillol.org/blog/index.php?Politique/2006/04/01

$24 \mathrm{http} / / / \mathrm{www}$.forum-algerie.com/parlons-en/27312-pourquoi-les-algeriens-begayent-quand-ils-parlent-en-arabe$\underline{\text { litteraire-41.html }}$

25 http://scripteur.typepad.com/corsicapolar/2009/02/quelque-part-entre-chyoda-petaouchnok-et-la-corsepar-ugopandolfi.html

${ }^{26} \mathrm{http}$ ://bebs-a-osaka.over-blog.com/categorie-1101613.html

${ }^{27}$ http://aerin.over-blog.com/article-no-more-pain-de-kat-tun-clip-52203624.html

${ }^{28} \mathrm{http}: / / w w w . f r a n c e s o i r . f r / s o c i e t e / 2008 / 11 / 13 /$ vivent-les-chauves.html

${ }^{29}$ http://jiminix.free.fr/?p=747 